LENGUA Y LITERATURA VASCA 



\title{
La estandarización del euskera
}

\author{
ARANTZAZU FERNÁNDEZ IGLESIAS \\ UNED \\ fernandezarantzazu@flog.uned.es \\ Recibido: mayo de 2012 Aceptado: junio de 2012
}

Resumen: La estandarización del euskara es un fenómeno reciente. El texto pretende explicar por qué el euskara batúa es el resultado de un proceso lento, controvertido, rápido y exitoso.

Palabras clave: euskara, estandarización lingüística, País Vasco

\begin{abstract}
The standardization of Basque language is a recent phenomenon. The text aims to explain why euskera batua is the result of a late, controversial but also fast and successful process.
\end{abstract}

Key words: euskera, language standardization, Basque Country

Se me ha invitado a hablar sobre el proceso de la estandarización del euskera, es decir, de la conformación del euskera batúa o unificado, en el contexto del congreso dedicado a la estandarización del sardo en el ámbito comparativo de las lenguas iberorromances ( $S$ 'istandardizatzione de su sardu in su cunfrontu cun sas limbas iberoromanzas). Antes de abordar el tema propiamente dicho deseo explicar algunos aspectos generales relativos a la lengua vasca y aclarar los límites de lo que voy a exponer aquí.

Una descripción somera del entorno geográfico y administrativo de la lengua vasca así como de la historia de sus modelos de lengua literaria ${ }^{1}$ en relación con las variantes dialectales nos permitirán comprender mejor cuáles han sido y son las dificultades que entrañan tanto el proceso de estandarización de la lengua como su recepción y difusión en la comunidad lingüística a la que se ha dirigido.

${ }^{1}$ La debilidad del sistema literario vasco lleva a incluir en su canon obras que no son estrictamente literarias y sería más propio hablar de modelos escritos. Sin embargo mantenemos la expresión de «modelos literarios» por ser la de más amplio uso entre las referencias bibliográficas del tema. 
Tampoco está de más recordar que aunque hay sólidas razones para considerar el proceso de estandarización como prácticamente consolidado éste prosigue desarrollándose en el contexto de una nueva era lingüística caracterizada por el uso de Internet, el multilingüismo, la preeminencia del inglés y la previsión de la desaparición de unas 3000 lenguas en este siglo, factores que han transformado la ecología lingüística mundial. (Crystal 2004: 16-17).

Y finalmente una última puntualización. Se suele presentar el caso de la recuperación del euskera - en el que la lengua unificada ha sido una herramienta imprescindible en el estricto sentido del término- como un ejemplo de inversión del peligro de desaparición de una lengua. Aunque hay datos que así lo corroboran, hay otros que apuntan a sus evidentes carencias y sus llamativas debilidades. Los datos que aportan las encuestas sobre el uso y conocimiento del euskera, sobre su normalización, son dispares en virtud de la zona que analicen, sin embargo la implantación de la lengua común ${ }^{2}$, su adopción como forma culta del euskera queda fuera de toda duda en cualquier condición y/o territorio.

El proceso de su creación y aplicación puede ser calificado como tardío, controvertido, rápido y exitoso y eso es lo que vamos a tratar de explicar aquí.

\section{CUESTIONES GENERALES: LÍMITES GEOGRÁFICOS, FRAGMEN- TACIÓN DIALECTAL, DISPERSIÓN ADMINISTRATIVA.}

Es comúnmente conocido que el euskera, lengua preindoeuropea considerada la más antigua de Europa, ocupó un territorio que a través de los siglos fue reduciéndose frente a la expansión del latín, el francés y el español. La falta de administración que la amparase, su status de lengua no oficial, las prohibiciones sobre su uso en el franquismo son factores cuyas consecuencias se iban traduciendo en una pérdida continua de hablantes que comienza a invertirse a finales de la década de los sesenta del siglo XX.

A ambos lados de los Pirineos la presencia de la lengua vasca arcaica (circa año 0) se extiende hasta la frontera del Garona por el norte, el valle de Arán por el este, la actual localidad de Laredo por el oeste y ya en el sur, territorios que abarcaban los enclaves riojanos de Haro, Logroño, las antigua Calagurris (Calahorra) y la ciudad de Turiasu (hoy Tarazona) y Caesaraugusta.

Según K. Cid (2002:24) en la zona peninsular la bibliografía documenta una pérdida continua de territorio con dos momentos de excepción: la caída del Imperio Romano con la subsiguiente ruralización que permitió la ampliación de los límites del euskera (Rioja Alta y Burgos) y la llamada Reconquista en la que hubo una importante repoblación vasca de las tierras de la Rioja y Burgos. La in-

2 Para la denominación del euskera estándar se utilizarán a lo largo de la exposición indistintamente los términos euskera batúa, lengua literaria común, lengua culta o lengua unificada. Su principal característica ha sido que, además de ser el resultado de un acuerdo y de la reglamentación de las convenciones subsiguientes, es un modelo de lengua que en su primera etapa de formación en manos de Euskaltzaindia se refiere a la norma escrita (Villasante 1980). Hoy en día esa afirmación no sería totalmente exacta. 
corporación del reino de Navarra al de Castilla oficializaría y reforzaría el uso del castellano en lugar del navarro-aragonés y cuando dicho dialecto cayó bajo el peso del castellano, el proceso de pérdida del euskera adquirió un ritmo imparable

En la zona vasca continental (la actual zona vasco-francesa) los límites del euskera se mantuvieron prácticamente estables durante 500 años, hasta que a finales del siglo XIX la transmisión de la lengua comenzó a decaer debido, entre otros factores, a la presión del francés, que también arrastró a los dialectos occitanos, y a un desarrollo económico que convirtió esos territorios en tierra de turismo y emigración ${ }^{3}$.

La dialectalización de la lengua objeto de esta comunicación es otro de los rasgos que merece la pena ser recordado aquí. Louis Lucien Bonaparte (18131891), atraído por la singularidad de la lengua vasca, visitó el País Vasco entre los años 1856 y 1869 en cinco ocasiones en las que desarrolló sus investigaciones como vascólogo junto con un grupo de colaboradores de la zona. Bajo su mecenazgo se publicó en 1863 el primer mapa científico de los dialectos vascos, «Cartes des Sept Provinces Basques montrant la délimitation actuelle de l'Euskera et sa division en dialectes, sous-dialectes et variétés» y el estudio del verbo «Le verbe basque en tableaux» publicado tres años más tarde, donde analiza las formas verbales vascas en ocho dialectos. La clasificación de las hablas del euskera que se siguió de sus estudios ha sido canónica en el campo de la filología vasca hasta que en 1998 Koldo Zuazo publica en el artículo Euskalkiak gaur [Los dialectos hoy] el mapa de referencia actual de la descripción de los dialectos del euskera.

Bonaparte clasificó el euskera en 8 dialectos, 25 subdialectos y casi 50 variedades de los mismos que reagruparía en tres bloques:

1. Vizcaíno

2. Dialectos centrales: Guipuzcoano, Labortano, Altonavarro septentrional y Altonavarro meridional

3. Dialectos Orientales: Suletino, Bajonavarro oriental, Bajonavarro occidental

Zuazo presenta en su mapa la siguiente clasificación zonal:

1. Dialecto occidental (vizcaíno)

2. Dialecto central (guipuzcoano)

3. Dialecto navarro

4. Dialecto navarro-labortano

5. Dialecto suletino

6. Colorea asimismo las zonas hispanófonas que eran vascófonas en el siglo XIX (según el mapa de L.L. Bonaparte).

${ }^{3}$ Entre 1876 y 1975, en la costa, la población pasa de 152.000 a 230.000 (aumento de un 40\%) pero en el interior se pasa de 78.000 a 55.000 (pérdida del $42 \%$ ). La costa pasa de 58.000 a más de 150.000. El éxodo rural de la zona interior no se ha detenido hasta la actualidad y los municipios no cesan de perder habitantes. 
La diversidad que se observa entre los dialectos (léxico, morfología verbal y distintas realizaciones fonéticas) viene acompañada de la idea de que los dialectos de los bordes del mapa lingüístico son más frágiles, están más expuestos a la absorción y contaminación lingüística a la par que alejados de la mayor homogeneidad de las hablas del centro. Se califican a menudo como de más irregulares, más localistas y más difíciles de comprender. Además, como veremos más adelante, el cultivo de la literatura se desarrolla en cuatro dialectos distintos que se relevan en el status de lengua literaria. Por esta razón aquellos que apostaban por elegir como lengua común la variante prestigiada por la tradición literaria, podían optar entre al menos cuatro de certificado prestigio. En este sentido K. Mitxelena había descrito en el Plan base o Proyecto General que le encomendó redactar Euskaltzaindia para las jornadas de Aránzazu en 1968 que

Hoy por hoy, al menos, en cualquier caso todos tendremos que ceder, quien más quien menos si se quiere alcanzar la meta. (...) Hay que reconocer, no obstante, que el euskera en su estado actual no constituye un sistema sino un diasistema ${ }^{4}$. Mitxelena in Villasante (1980: 49)

La unanimidad por parte de los estudiosos implicados en el proceso de estandarización fue en algunos momentos difícil de alcanzar debido a susceptibilidades que se sentían marginadas si se elegía una u otra variante del euskera literario.

Los casi 700.000 hablantes del euskera o vasco se reparten en dos países: Francia y España y en tres Administraciones distintas: La Comunidad Autónoma Vasca (CAV) que engloba tres provincias, Álava, Bizkaia y Gipuzkoa, la Comunidad Foral de Navarra (CFN), y la parte occidental del Departamento de Pirineos Atlánticos francés en el que están agrupadas las provincias de Labourd, la Baja Navarra y la Soule. La población total de esos territorios es de 3.114.276 habitantes según los últimos datos oficiales correspondientes a 2011. Además se calcula en unos 100.000 el número de euskaldunes (vascoparlantes) en la diáspora.

El status legal de la lengua no coincide en los territorios citados como no coinciden las políticas lingüísticas de las Administraciones a las que hemos hecho alusión. En la zona peninsular es lengua oficial junto con el español en la $\mathrm{CAV}$, pero en la CFN lo es sólo en una de las tres zonas lingüísticas en las que se divide la provincia (zona vascófona, mixta y no vascófona); así las cosas, sólo en la zona definida como vascófona la Administración tiene la obligación de atender en euskera. En los territorios bajo la Administración francesa englobados en el Departamento de los Pirineos Atlánticos de la región de Aquitania, la única lengua oficial es el francés. Es fácil deducir, por tanto, que la salud del euskera, lengua minoritaria bajo dos Estados y tres Administraciones locales dis-

${ }^{4}$ La traducción del texto original es de Villasante, quien asimismo indica en una nota a pie de página que el vasco con sus cuatro dialectos literarios no constituye un sistema sino cuatro (o más) sistemas literarios. 
tintas, no es homogénea, como no lo es el respeto a los derechos lingüísticos de sus hablantes.

Como hemos señalado, en el último cuarto del siglo XX se certifica la inversión del fenómeno de la pérdida de hablantes; los instrumentos de esa certificación son las encuestas sociológicas del Gobierno Vasco realizadas desde 1996 a la población de 16 años en adelante que permiten contrastar información de todos los territorios. La V Encuesta Sociológica (Gobierno Vasco 2012) arroja los siguientes datos:

Entre los habitantes de 16 años o más, hay 185.600 personas bilingües más en 2011 que en 1991 (en la actualidad constituyen un 27\%). Este aumento de la población bilingüe ha tenido lugar sobre todo en la Comunidad Foral de Navarra y en la CAV. En el País Vasco Norte, si bien continúa habiendo pérdidas, éstas se han ralentizado, y continúa creciendo el número de jóvenes bilingües. Al mismo tiempo, entre las personas de 16 años, en la totalidad de los territorios hay, en la actualidad, 140.239 erdaldunes menos que hace 20 años (han pasado del 70\% en 1991 al 58\% en 2011). En la CAV como en la Comunidad Foral Navarra ha descendido en número de erdaldunes. Por el contrario, en el País Vasco Norte estos han aumentado tanto en términos absolutos como en porcentuales ${ }^{5}$.

El porcentaje de bilingües pasivos se ha duplicado en el conjunto del territorio en los últimos 20 años, es decir, hoy en día representan el 14,7\%, mientras que en 1991 suponían el 7,7\%. El crecimiento más alto se ha producido en la CAV y en Navarra, y en menor medida, en el País Vasco Norte.

Los datos referidos a la competencia lingüística por territorios se desglosan de la siguiente manera:

\begin{tabular}{|l|l|l|l|l|}
\hline & Todos los territorios & CAV & Navarra & Norte \\
\hline Total & $\mathbf{2 . 6 4 8 . 9 9 8}$ & $\mathbf{1 . 8 7 3 . 4 9 8}$ & $\mathbf{5 3 7 . 1 3 9}$ & $\mathbf{2 3 8 . 3 6}$ \\
\hline Bilingües & $\begin{array}{l}714.136 \\
(27 \%)\end{array}$ & $\begin{array}{l}600.058 \\
32,0 \%^{6}\end{array}$ & $\begin{array}{l}62.977 \\
11,7 \%^{7}\end{array}$ & $\begin{array}{l}51.100 \\
21,4 \% \%^{8}\end{array}$ \\
\hline Bilingües pasivos & $\begin{array}{l}388.255 \\
(14,7 \%)\end{array}$ & 325.967 & 40.546 & 21.742 \\
\hline Erdaldunes & $\begin{array}{l}1.546 .607 \\
(58,4 \%)\end{array}$ & 947.473 & 433.616 & 165.518 \\
\hline
\end{tabular}

${ }^{5}$ Aclaramos un par de términos: erdaldunes, aquí monolingües castellanos; País Vasco Norte, denominación común de los territorios vascos continentales sitos en Francia.

${ }^{6}$ En 1991 el porcentaje de bilingües era $24,1 \%$ en la CAV.

${ }^{7}$ En 1991 el porcentaje de bilingües era el 9,5\% EN LA CFN.

${ }^{8}$ En 1996 el porcentaje de bilingües era de 26,4\% y 22,5\% en 2006 en el País vasco-francés (figura como Norte). 
Los datos referidos al uso del euskera en el conjunto de los territorios se desglosan de la siguiente manera:

\begin{tabular}{|l|l|}
\hline Uso en el conjunto de los territorios & total \\
\hline Utiliza el euskera en mayor o menor medida & $24,2 \%$ \\
\hline Más que el castellano & $16,1 \%$ \\
\hline Más que el francés & $8,1 \%$ \\
\hline
\end{tabular}

El porcentaje del uso intensivo -en mayor medida que el francés o el castellano- se distribuye por territorios de la siguiente manera:

\begin{tabular}{|l|l|}
\hline Uso intensivo del euskera & total \\
\hline CAV & $20 \%$ \\
\hline País Vasco Francés & $9,6 \%$ \\
\hline Navarra & $5,5 \%$ \\
\hline
\end{tabular}

El aumento del número de bilingües que señalan las encuestas entre el año 1991 y 2011 se cifra en 185.600 bilingües más. Este incremento ha sido posible gracias a los programas de recuperación lingüística aplicados en la CAV que se pusieron en marcha desde la designación del euskera como lengua cooficial en el Estatuto de Autonomía aprobado en 1979 con la llegada de la democracia en España; junto con la subsiguiente Ley de Normalización y Uso del Euskera de 1982 posibilitaron la implantación de un modelo educativo que contemplara la enseñanza en euskera. Es también el caso de Navarra con la Ley Foral del Vascuence de 1986 que determinó la oficialidad del euskera en la zona vascófona de la Comunidad Foral Navarra (CFN) así como la ordenación de su enseñanza en la misma.

El euskera constituye una seña de identidad para la mayoría de los vascos que lo conocen y es sin lugar a duda, el aglutinador social más importante para los euskaldunes [vascoparlantes]. La asunción de su estandarización, particularmente en la CAV, reside en la confluencia de varios factores a partir de los años 60: la rapidez con que se produjo esa normativización del euskera común, una militancia social que demandaba y activaba medidas de defensa de la lengua por considerar que se trata de un bien común en peligro y una situación política propicia para las políticas lingüísticas que fomentaran su uso. P. Baztarrika (2010:143) no duda en afirmar que el factor más relevante es el de la voluntad del hablante, la conducta y la adhesión del hablante.

El empuje que se ha dado a su conocimiento hubiera sido imposible si no se hubiera contado con un modelo de lengua unificado aceptado como vehículo para la expresión escrita especialmente en el campo de la enseñanza, los medios de comunicación y la Administración, pero nunca hay que dejar de señalar que la mejora en la situación del euskera se debe a la voluntad y al esfuerzo social que 
comenzó en los años 60 bajo el signo de la urgencia ante los cambios demográficos que se advertían debidos a la industrialización. El euskera lo unificaron los expertos lingüistas pero su aplicación vino en un primer momento de la mano de la red de gaueskolas e ikastolas ${ }^{9}$ y desde 1982 de todos aquellos profesores y alumnos, funcionarios de la administración, periodistas, traductores etc. que aprendieron la lengua o se alfabetizaron en ella. Estas gentes constituyen un movimiento que arropó el código de los lingüistas en un intento - esta vez socialmente común- de paliar el retroceso de la lengua.

Así las cosas, si aplicamos la metodología propuesta por el grupo de expertos de la ONU en el documento Vitalidad y peligro de desaparición de las lenguas $(2003)^{10}$ hemos de colegir que la lengua vasca ya no corre peligro de desaparecer porque cumple condiciones socio-históricas que la eximen de figurar en la lista de las lenguas en peligro de extinción [endangered languages]. Contamos con una cantidad de hablantes que sobrepasa con creces la cifra de 100.000 y la CAV y la CFN , están dotadas de la infraestructura política necesaria para el posible desarrollo de políticas de apoyo a la lengua. La transmisión intergeneracional en éstas está garantizada así como su presencia en el sistema educativo. Además la sociedad vasca cuenta con un sistema cultural y/o literario suficientemente desarrollado que garantiza de manera aceptable -aunque mejorable- la producción, mediación, recepción y recreación de la literatura en euskera.

Sin embargo, aunque el miedo a la desaparición del euskera parece haberse diluido, hay señales que indican que su situación es susceptible de ser fragilizada fácilmente porque depende en exceso de los avatares políticos que determinan una mayor o menor orientación de las Administraciones hacia medidas de amparo y protección. La diferencia administrativa que hemos mencionado al principio conlleva aparejada una distinta salud para cada territorio:

${ }^{9}$ Gaueskolas: centros para adultos dedicados a la enseñanza y/o alfabetización en euskera; ikastolas: escuelas en las que se estudia en euskera cuyo origen se remonta a la II República. Sus actividades fueron prohibidas durante el franquismo.

${ }^{10}$ Una lengua corre el peligro de desaparecer cuando sus hablantes dejan de utilizarla, cuando van restringiendo su uso a ámbitos cada vez más reducidos, cuando recurren cada vez menos a sus registros y estilos idiomáticos, o cuando dejan de transmitirla a la generación siguiente. Un solo factor no es determinante para afirmar que una lengua se halla en peligro de desaparición. Los expertos de la UNESCO han identificado los nueve factores siguientes, que deben considerarse en su conjunto en el documento Vitalidad y peligro de desaparición de las lenguas adoptado en la reunión internacional de expertos del programa Salvaguardia de las Lenguas en Peligro, celebrada en París 2003:

La transmisión del idioma de una generación a otra, el número absoluto de sus hablantes, la proporción de sus hablantes con respecto a la población total, los cambios en los ámbitos de utilización del idioma, la capacidad de reacción ante los nuevos ámbitos de actividad y los media, la disponibilidad de material destinado a la alfabetización en el idioma y la enseñanza de éste, la actitud ante el idioma y la política lingüística de las autoridades gubernamentales y las instituciones, comprendida la cuestión de su reconocimiento y uso oficiales, la actitud de los miembros de la comunidad de hablantes hacia su propio idioma, la cantidad y calidad de documentos en el idioma. 
La distribución de los bilingües actualmente es más densa en la zona peninsular y en concreto en la provincia de Gipuzkoa, lo que convierte a esta provincia en el foco de irradiación cultural más claro. Sin embargo, incluso allí el euskera no llega a estar presente en todos los ámbitos de la Administración. En Navarra la política de las instituciones autonómicas es observada por muchos estos últimos años como contraria a la defensa de los derechos lingüísticos de los vascoparlantes y, por último, el territorio vasco-francés presenta una situación de fragilidad francamente preocupante porque -a pesar del esfuerzo militante de sus hablantes- su número de locutores está en la frontera de $\operatorname{los} 80.000^{11}$ y carece del apoyo de la Administración por tratarse de una lengua regional según el ordenamiento francés.

\section{LA ESTANDARIZACIÓN DE LA LENGUA}

La estandarización de la lengua vasca debe ser explicada desde dos maneras que se siguen en el tiempo.

Los primeros pasos hacia una lengua común los constituyen los esfuerzos de los autores anteriores al siglo XX quienes- ante la ausencia de instituciones políticas o académicas que intentaran establecer un modelo de lengua, procuran escribir en un euskera que fuera comprensible para el mayor número de vascos posible. La preocupación por una lengua común era lógica porque las letras vascas eran una herramienta al servicio del proselitismo de la Iglesia ${ }^{12}$.

En segundo lugar contamos con las ideas en torno a la defensa del euskera que se abren paso en los años finales del XIX como una reivindicación del naciente nacionalismo vasco y que se encauzarán -no fácilmente en un principio- en las propuestas de estandarización de Euskaltzaindia, la Real Academia de la Lengua Vasca. Ésta es la institución clave en el proceso de unificación y regulación del euskera que culmina en la adopción del «euskera batúa» (euskera unificado) como resultado de la firme y mayoritaria convicción social de que esa lengua culta/literaria/ regulada y unificada era el único camino para salvar a una lengua que se consideraba en peligro de desaparición. Así lo expresaba K. Mitxelena:

El hacer entrar al euskera por el camino que conduce a la unificación, lo consideramos imprescindible, absolutamente necesario para la vida de la lengua. Mitxelena in Villasante (opus cit. 1980: 48)

La cuestión era cómo. ¿Optando por la adopción de uno de los dialectos con tradición literaria? ¿Optando por el dialecto con el mayor número de hablantes? ¿Construyendo una base común a todos los dialectos?

\footnotetext{
${ }^{11}$ El último Atlas de las lenguas del mundo en peligro de la UNESCO (Moseley, 2010) no desglosa el territorio y presenta el euskera como lengua vulnerable pero no amenazada [endagered]. Sin embargo puntualiza severely endangered in France with fewer than 80.000 speakers.

${ }^{12} \mathrm{El} 90 \%$ de los autores en lengua vasca de los siglos XVI, XVII y XVIII eran sacerdotes (Sarasola, opus cit. 52)
} 


\subsection{Hacia la lengua común: el camino de la literatura}

El primer libro en lengua vasca, Linguae Vasconum Primitiae, se editó en Burdeos el año 1545 al amparo de la invención de la imprenta y en vísperas del Concilio de Trento. En este texto fundacional de la literatura vasca hay una conciencia lingüística manifiesta que llama al orgullo de dejar demostrado que el euskera, en contra de la opinión de algunos, es una lengua que puede ser escrita y tiene, por tanto, el mismo rango que otras lenguas europeas tales como el italiano, francés o español.

Heuscara Ialgui adi plaçara
Berce gendec vste çuten
Ecin scriba çayteyen
Oray dute phorogatu
Enganatu cirela.

Heuscara

Ialgui adi mundura ${ }^{13}(\ldots)$

La composición dice Heuscara/ Ialgui adi mundura [¡Euskera, sal al mundo!] en uno de los dos poemas escritos en alabanza de la lengua vasca por Bernard Etxepare, párroco de Eyheralarre, en la Bajanavarra. Éste escribió un texto compuesto por -además de los poemas dedicados a la lengua vasca, por poemas erotico-amorosos y religiosos- en el dialecto bajonavarro, es decir, utilizó la modalidad de sus feligreses y la suya propia. Digamos que en términos de hoy en día, dio una solución local para un problema global. Desde la conciencia lingüística de quien sabe que ha puesto en marcha la letras vascas instó a la difusión de las mismas desde sus versos pero la lengua que utilizó, el bajonavarro de corte popular, no tuvo continuadores porque el autor que tomó el relevo de literario utilizó otra variante del idioma.

Cuando en 1571 se publicó Jesus Krist Gure Jaunaren Testamentu Berria [El Nuevo Testamento de Nuestro Señor Jesucristo] contenía una reflexión sobre el modelo de lengua por el que había optado su autor, el labortano Joannes Leizarraga (1506-1601) ${ }^{14}$. En el prólogo titulado Hescualduney [A los vascos] se dirige a sus lectores explicando que bat bederac daqui heuscal herrian quasi etche batetic bercera-ere minçatzeco manerán cer differentiá eta diuersitatea den [Cualquiera sabe lo diferente y diversa que es la forma de hablar en Euskal Herria casi de una casa a otra] y añade que ha seguido el criterio de, sin cambiar el sentido original, seguir un lenguaje que sea comprensible por la mayoría, sin caer en localismos.

\footnotetext{
${ }^{13}$ Traducc.: Euskera sal a la calle/ Otras gentes creían/ Que no era posible escribirte/ Ahora han comprobado/ que estaban engañados.// ¡Euskera sal al mundo!

${ }^{14}$ Había recibido el encargo del sínodo calvinista de Pau (1564) para que tradujera la Biblia como instrumento del protestantismo al que se había convertido la corte navarra bajo el reinado de Joanna d'Albret.
} 
(...) razoin hunegatik sensu egiazkotik aldaratu gabe, lengoajeaz den bezenbatean ahalik gehiena guziei adi eraziteari iarreiki izan gaitzaitza, eta ez xoil edozein leku iakineko lenhoaje bereziri ${ }^{15}$.

Leizarraga era consciente de que debía utilizar una lengua accesible (ahalik gehiena guziei adi eraziteari iarreiki izan gaitzaitza) para sus propósitos y para ello no se adscribe exclusivamente a su dialecto labortano. De hecho, la lengua a la que vertió el Nuevo Testamento es considerada la primera koiné vasca, una lengua de base labortana en la que se observan rasgos de los otros dialectos continentales: el bajonavarro y el suletino. Su lengua es excesivamente arcaizante, reverencial, con abundantes préstamos del latín y del griego como resultado de su apego a un texto «sagrado» del que desea desviarse lo menos posible. Su modelo no prosperó por el triunfo de la Contrarreforma.

Es en el siglo XVII cuando nace el labortano clásico como modelo de lengua consolidado y adoptado por los escritores del otro lado de los Pirineos. El euskera que el clérigo Axular (Pedro de Aguerre y Azpilicueta 1556-1644) utilizó como vehículo de su libro de ascética Gero es el paradigma de lengua clásica que fue adoptada por los miembros de la Escuela de Sara. En este pueblecito de la provincia de Labourt se reunía un grupo de religiosos escritores bajo la tutela intelectual del párroco de la localidad. El labortano clásico se consagra como una variante de prestigio que sería postulada por intelectuales del siglo XX como la opción para la lengua literaria común.

Cuando en el siglo XVIII el centro de la producción literaria bascula desde Lapurdi hacia el País Vasco peninsular son los escritores guipuzcoanos los que consagran literariamente su dialecto. El jesuita guipuzcoano Manuel de Larramendi (1690-1766) autor de la gramática El Imposible vencido Arte de la lengua Bascongada y del Diccionario Trilingüe (castellano-euskera-latín) es el impulsor del movimiento que convirtió Gipuzkoa en el foco principal de las letras vascas. El empuje del mismo arrastra a que escritores vizcaínos y navarros también lo adopten. Sería por tanto la segunda vez con la que contamos con un dialecto consagrado como lengua culta y por tanto como modelo de lengua que cumple la tarea de lengua unificada.

El dialecto vizcaíno tendrá su momento de esplendor en el siglo XIX cuando Pedro Antonio Añibarro (1748-1830) y Joan Antonio Mogel (1745-1804) por otro produzcan sus obras en dos modelos lingüísticos diferentes: el vizcaíno de Arratia y el de Markina respectivamente. Hasta la adopción del euskera batúa son estos dos modelos los que han sido utilizados por los escritores que optaban por escribir en vizcaíno.

Con respecto a esta variante de la lengua las ideas de Sabino Arana (18651903), fundador del EAJ/PNV, y el manual de ortografía que redactó, Lecciones de ortografía del euskera bizcaino (1896) gozaron de un predicamento que excedía lo

15 Traducc.: Por esta razón, sin cambiar su verdadero sentido seguimos un lenguaje que sea por lo menos comprensible por la mayoría y no un lenguaje conocido unicamente en un lugar particular. 
puramente lingüístico. De hecho, cuando triunfen las propuestas de estandarización de Mitxelena y Villasante en los años 60, la vieja guardia nacionalista no será nada proclive a abandonar la idea de un vizcaíno regulado que era la que ellos defienden en aquel momento. En la base de esta concepción se halla el proyecto político de Arana, una federación de tierras vascas en las que cada una de las siete provincias tuviera un alto grado de autonomía. No se contemplaba desde el nacionalismo de estirpe sabiniana el euskera unificado como algo propio y sus miembros se constituyeron en un grupo de presión que dificultó el camino hacia la estandarización en nombre de la defensa de la pureza de la lengua.

\subsection{Hacia la lengua común desde la institución académica: Euskaltzaindia}

\subsubsection{Los años anteriores a la guerra (1920-1936)}

Euskaltzaindia, la Real Academia de la Lengua Vasca, es la institución desde la que se ha formalizado el euskera unificado. Fundada en 1919 por las diputaciones del País Vasco reunía a representantes de las siete provincias vascas. En su documento fundacional declaraba expresamente que emprendería la tarea de un modelo [de lengua] a partir de [la sangre] de todos los dialectos del euskera para el uso de todos los vascoparlantes:

Ekingo du euskalgi oroen odolez azitako euskal-eredu bat gertutzen, itzez, itz- yoskeraz ta idazkeraz albait erabatetsuena, euskaldun guziak idatz-irakurketan ${ }^{16}$

(Euskaltzaindiaren Sortarauak 6. artikulua, 1920:3)

La tarea, sin embargo, no fue nada fácil porque si bien existía acuerdo sobre la necesidad de contar con un euskera normativizado y común, no hubo hasta bien entrados los años sesenta, un planteamiento que fuera aceptado de manera unánime por los miembros de la institución. El problema era no sólo desde qué punto de partida comenzar sino la concepción, en la práctica, del euskera como una manifestación no mucho más allá que folclórica de la cultura vasca entre los miembros de la Academia.

Para hacernos una idea de la disparidad de pareceres y la dispersión de fuerzas contaremos a modo de ejemplo que a instancias del informe que Arturo Campión (1854-1937) y Pierre Broussain (1859-1929) presentaron en el año 1922, se organizaron cuatro comisiones que habrían de presentar sus propuestas de unificación. Se defendieron 16 informes distintos de los que 5 eran contrarios a la unificación.

${ }^{16}$ Traducc.: Acercará un modelo de vasco alimentado de la sangre de todos los dialectos, lo más unificado posible en sus palabras, pronunciación, en la lectura y escritura de todos los vascoparlantes. 
Uno de los informes, bajo el purismo lingüístico que buscaba la originalidad en lo primigenio y trataba de limpiar la lengua de influencias extranjeras, proponía el jatorrizko euskera [el euskera original], es decir, una lengua desprovista de evolución. Otro de ellos, el defendido por Agustín Anabitarte (1891-1981), se adhería a la propuesta de unificar la lengua desde el guipuzcoano o desde el labortano. Además otra propuesta se decantaba por la variante vizcaína, otras dos por la guipuzcoana (giputza) y dos más por el gipuzkera osatua [guipuzcoano completado] de R. M. Azkue (1864-1951). Finalmente otras dos propuestas tomaban posición a favor del euskera osatua (euskera completado), es decir, un euskera unificado conseguido de la mezcla de todos los dialectos.

Mientras tanto los académicos se expresaban en español en sus reuniones y redactaban en guipuzcoano los documentos públicos conjuntos (Euskera 32, 922:11). Los caminos de la unificación divergían y las opiniones ajenas a Euskaltzaindia tampoco parecían ayudar mucho. Así decía Menéndez Pidal (1962: 11-57) en conferencia que pronunció en Bilbao en los años 20 acerca de la unificación:

Hay que partir del principio de que el lenguaje es ante todo un producto social, colectivo, un fenómeno natural que está por encima de los artificios convencionales de los gramáticos. Podrá la humanidad llegar a hablar una lengua única en siglos lejanos, pero no llegará a esa unificación inventando volapukes, esperantos idos y demás mezclas de todos los idiomas. Podréis llegar a tener un eusquera literario único, pero eso no será obra momentánea. Fácil es relativamente hacer un libro para el uso de unos cuantos escritores, una combinación del guipuzcoano y labortano y proveerla de perfecciones de otros dialectos; pero esa creo yo no llegará a ser la lengua del pueblo. (La cursiva es mía.)

\subsubsection{Los años posteriores a la guerra: 1945-1960}

La guerra civil española y la dictadura que le siguió supuso el exilio y la cárcel para la mayoría de los intelectuales vascos. El euskera fue severamente reprimido y prohibido por los franquistas en los años posteriores a la contienda (Torrealdai 1998).

Con el devenir del tiempo, en la década de los 60, la sociedad vasca peninsular se trasformó en una sociedad que avanzaba hacia la modernidad gracias a la industrialización que atrajo un importante número de emigrantes de otras zonas de España. En el País Vasco francés la mano de obra deja de emigrar a América para hacerlo a las ciudades del interior de Francia: París, Burdeos Pau; a la vez, el eje costero Anglet-Biarritz-Bayona adquiría una fuerza comercial y turística que no era capaz de frenar la despoblación del interior.

El empuje al euskera se sostiene de la mano de la revista Euzko-Gogoa fundada en 1950 en Guatemala. Desde ella se defendía y practicaba la idea de 
la lengua vasca como una lengua de cultura en los nueve años en los que fue editada.

No todos opinaban lo mismo: el escritor Nikolas Ormaetxea Orixe (18881961) asociaba la lengua vasca a un uso poético en pos de la belleza y alejada del ámbito de la ciencia porque en su opinión las científicas no eran lenguas originalmente humanas.

Sin embargo, aunque una vez más cada cual a su manera, se retomaba la idea de una lengua común que sirviera para los usos escritos, de una lengua de cultura que convirtiera -en palabras de Mitxelena- la larre hizkuntza [lengua del campo] en una kale hizkuntza [lengua de ciudad].

El planteamiento hasta los 60 vuelve a ser parecido al de antes de la guerra, la tradición literaria marca la preferencia de unos y otros por uno u otro dialecto y la propia Euskaltzaindia no es capaz de ir más allá de redactar unas cuantas reglas ortográficas. Las dificultades en el avance hacia el modelo de lengua estándar son una característica de este periodo.

Entre las figuras de la época destaca la del getxotarra Federiko Krutwing (1921-1998) quien defendía la tesis de que el labortano antiguo (lapurtera zaharra), la lengua de Leizarraga, era la apropiada para ser la lengua culta de los vascos. En realidad hay que reconocerle el mérito de haber activado a los académicos en el camino de la unificación pero su propuesta estaba lejos de ser esa lengua vivida y cercana que se ha de priorizar en el camino de la unificación si es que se desea que ésta se extienda. La lengua de Leizarraga no dejaba de ser una lengua que nunca se había utilizado por los hablantes y a la que escritores como G. Aresti (1933 - 1975) o J. Mirande (1925-1972) se adscribieron pero tan solo en un primer momento.

Los escritores de la zona francesa escribían tras el declive del labortano costero en el siglo XIX, en una variante llamada navarro-labortana cuyos exponentes mejor conocidos son las revistas Euskualduna y Gure Herria. Se trataba de una forma de lengua que fue descalificada por P. Laffitte (1901-1985) como demasiado popular.

El desarrollo del gipuzkera osatua [guipuzcoano completado] inclinó sus características hacia los localismos guipuzcoanos lo cual creó el rechazo de los vizcaínos que lo había defendido como la lengua de su producción escrita y dejó de ser considerado una opción universal.

En euskera osatua [euskera conpletado] fue escrita la obra Kurloiak [Gorriones] publicada por José Basterretxea, Oskillaso, en 1962, un texto que servía de ilustración práctica a los principio de este modelo lingüístico y que gozó de cierto éxito. En el resto de obras que se vehicularon en esa modalidad de euskera unificado, la lengua adolece de artificiosidad, de falta de coherencia a la hora de elegir las variantes del léxico y de una utilización sin rigor de las formas vecinas que lo convirtieron en una lengua de nadie.

Así las cosas, en la década de los 50, Euskaltzaindia que había sido fundada en 1918, sólo había determinado: 
- en 1953

- Se aceptan las grafías $<\mathrm{dd}>,<\mathrm{ll}>,<\mathrm{rr}>,<\mathrm{tt}>$ en lugar de la tilde sobre la consonante.

- La conservación de la ortografía original en topónimos y antropónimos extranjeros.

- La utilización de la $<\mathrm{h}>$ se permite en la zona peninsular entre vocales iguales (ahate/ohore/ zuhurra/ ) y como forma de evitar la homonimia (ori-hori/ura-hura/ari-ahari).

- No regula la palatización salvo el caso de baiño que se escribe así.

- en 1959

- Se aceptan como vascas palabras de origen latino (aingeru, arraza, eskola, familia, kantatu, abendu, por ejemplo) que habían sido rechazadas por los garbizales [puristas] de principio de siglo. Estos preferían crear palabras nuevas (gotzon, enda, ikastola, sendi, abestu, gabonil) antes de utilizar préstamos.

- Se aceptan palabras internacionales como politika, teknika, kilometro, literatura.

- Se aconseja mantener la sintaxis y la morfología sin alterar.

\subsubsection{A partir de los años 60}

El desarrollo industrial de los años sesenta viene acompañado de cierta apertura en la dictadura que posibilita la existencia de una eclosión cultural vasca militante y reivindicativa que asume la tarea de impedir la desaparición de la lengua y la identidad vasca que ella sustenta ${ }^{17}$. Son los años en los que surgen los grupos artísticos Gaur, Ez dok hamairu, y se renuevan las revistas Jakin, Zeruko Argia, Anaitasuna etc. A finales de los sesenta vuelve a ponerse en pie la red de ikastolas que se consolidan en la década siguiente y surgen las gaueskolas. En 1964 se funda en Bayona (Francia) el grupo Euskal Idazkaritza formado por representantes de ambos lados del Pirineo. Cuenta con una sección dedicada a la lengua, Hizkuntza Saila, desde la que reivindican desde el primer momento el euskera unificado como su prioridad y asumen la tarea de encaminarse hacia la misma cuanto antes. Ante la inoperancia de Euskaltzaindia que había denunciado Jose Álvarez Enparantza Txillardegi (1929-2012), preparan un documento de decisiones (publicado en la revista Jakin en 1965) que expondrán tras ocho meses de trabajo en una reunión abierta a la que acuden personas relacionadas con el euskera (activistas culturales, profesores...) de todas las zonas del País Vasco. Previamente los organizadores se habían asegurado el apoyo de las fuerzas nacionalistas del momento (EAJ, ETA, Enbata) y la Iglesia. Txillardegi da cuenta (2004:55-64) de las determinaciones tomadas, además de en el uso de la $\mathrm{H}$ en estas tres áreas:

\footnotetext{
${ }^{17}$ Justo al final de la década de los cincuenta surgieron Enbata (1956) y ETA (1958).
} 
- ortografía:

- a favor de las grafías $<\mathrm{x}>$, y la $<\mathrm{tx}>$

- Dobles consonantes $<\mathrm{tt}>,<\mathrm{rr}>,<\mathrm{ll}>$, $<\mathrm{tt}>$ en lugar de tilde

- Se destierra la $<\tilde{n}>$ a favor de la $<$ in $>$ para señalar la palatalización. Ej.: baino

- Se escribirá $<\mathrm{n}>$ ante $<-p>\mathrm{y}<-b>$. Ejs.: enparantza, gonbidapena.

- Se acepta la utilización de la h en los contextos de la regulación anterior (ahate, ori/hori) y se recomienda su adopción en una fase posterior para el principio de palabra (hartu, handi) y entre vocales distintas (ahizpa). No se admiten por buenas las grafías que reflejan la aspiración con las oclusivas: $<\mathrm{ph}\rangle,<\mathrm{th}\rangle,<\mathrm{kh}\rangle$ (ephe, urthe, bekhatu) ni detrás de consonate líquida (alhaba, senhar, erhi). Adjuntaron una separata con la lista de palabras con $<\mathrm{h}>$.

- Se dan normas para la utilización de prefijos:

ba- : badaki, junto si es condicional, ba dakil ez daki, separado, si es afirmación

bait- se escribirá en una sola palabra junto al verbo al que acompaña pero se admiten dos variantes: baitnaiz y bainaiz.

- declinación:

- Se regula la forma - ek para el sujeto plural de verbo transitivo (frente a $-a k)$

- Se regula el caso del inesivo, nori plural: -ei (frente a -eri)

- Se regula el caso instrumental (-az, $-e z$, se admiten ambas frente a las formas -tzaz, -taz)

- Se regula el caso destinativo (-entzat frente a -endako)

- Se regula el caso prolativo (-tzat)

- Se admite -etatik (frente a -etarik) en el caso ablativo nondik

- Se prioriza la desinencia -ra (frente a-rat) en el caso nora o adlativo

- Se sancionan las formas -a(ren)gan, -arengandik, -arengana frente al uso de -(a)ren baitan, -ganat... en el caso norengana o adlativo compuesto

- en el verbo:

- Regularon solamente las formas del auxiliar IZAN y *EDUN del presente: naiz (frente a niz, naz) y dut (frente a dot/det...) pero se adoptaron para el plural formas guipuzcoanas como gera, zera, zerate.

Lo importante es que se había producido un relevo generacional que había inaugurado otra manera de trabajar, lejos de las susceptibilidades y disquisiciones vacuas que había colonizado hasta esta década el discurso de Euskaltzaindia sobre la unificación del euskera.

En septiembre de 1968 había prevista una reunión de la Academia para tratar - una vez más- el tema de la unificación. Tres meses antes se fundaba la Idazleen Elkartea [Asociación de Escritores] que fue convocada por la Gede- 
riaga Elkartea [Asociación Gederiaga] de Ermua (Bizkaia) a una reunión en la que se decidió apoyar el proceso de unificación adoptando las propuestas de Bayona $^{18}$.

En 1968 comenzaban a plantearse en Euskaltzaindia los actos conmemorativos del 50 aniversario de su fundación. En este contexto el poeta Gabriel Aresti recriminó a la Academia no haber cumplido con su trabajo (Aresti 1968:3).

(... ) Akademiaren urrezko eztaiak hospatzeko asmoak aipatu zirenean, jaiak eta jaiak ekarri ziren ahora. Orduan haserratu nintzen: «Gureak guztiak dira jai egunak. Eta astegunak noizko dira? Lanean aritzeko orduak ez al du oraindik gure atea jo? (...) Katalanen eredua ekarri nuen aipuetara, Ponpeu Fabraren lan miragarria. Hizkuntza batek ezin duela bizitza normal bat eraman, erregla eta lege zehatz batzuei ez badago loturik. ${ }^{19}$ (...)

Koldo Mitxelena es el miembro de Euskaltzandia al que se le encargará la elaboración de una propuesta para el euskera batúa que habrá de debatirse en Aránzazu. (Euskera 13, 1968:137-265). En ella defiende la idea de que la base del euskera unificado es el dialecto central fusionado con la tradición literaria labortana. Continuando el trabajo de la reunión de Bayona, Mitxelena amplía las recomendaciones en el campo del léxico al determinar que la Academia no podía rechazar término alguno vivo en favor de formas ya desaparecidas del habla. También estimó que se favoreciera la forma fonéticamente más antigua frente a la nueva en variantes tales como bekatu (no pekatu), arima (no anima). Entre variantes dialectales se daría prioridad a las centrales (arrain, berri) frente a arrai, barri. En el campo de la morfología dio por válidas las propuestas de Bayona aunque puntualizando que en el caso ablativo, la desinencia -etarik era válida, así como también lo era en el caso del inesivo la partícula baitan.

Sus propuestas suscitaron una fuerte reacción contraria entre los miembros de mayor edad en el momento en que Euskaltzaindia las aceptó. La regulación sobre la $<\mathrm{H}>$ fue la llave que abrió la caja de los truenos, y de esa caja salieron a la luz posturas encontradas e irreconciliables. Frente a los que defienden un euskera puro, sin contaminar y localista, hay extremistas que identifican el batúa con el marxismo, la lucha armada y la falta de valores cristianos, en definitiva con algo extranjero ajeno a la esencia vasca. Sirvan de reflejo de esa mentalidad que se oponía a la estandarización de la lengua dos párrafos escritos por el nacionalista tradicional Josu Arenaza en 1974:

\footnotetext{
${ }^{18}$ Ese año también (Jakin 1968, vol. 32 y 32) se apueba etorri naizl etor naiteke, la distinción entre la raíz verbal (etor, ekar...) y el participio (etorri, ekarri...) que se distingue según el modo de conjugación.

${ }^{19}$ Traducc.: (...) Cuando se mencionaron los deseos de celebrar las Bodas de Oro [de la Academia] no se mencionaba otra cosa que no fueran fiestas. En ese momento me enfadé: « Para nosotros todos los días son días de asueto. Y los días de labor, ¿para cuándo son? ¿No ha llamado a nuestra puerta la hora de trabajar? Mencioné el ejemplo de los catalanes, el admirable trabajo de Pompeu Fabra. Una lengua no puede tener una vida normal si no está ligada a reglas y leyes definidas.
} 
La h es un símbolo político aunque lo nieguen muy sesudos señores, aunque vayan vestidos de seráficos hábitos. Tan político como puede ser la hoz y el martillo, la cruz gamada o las dos a la vez. Hemos mantenido la tesis de que la ortografía achista no es más que el intento de politizar el euskera, rompiendo los moldes tradicionales, como una rama más de lo que se intenta romper: la familia, el derecho a la educación cristiana, nuestras costumbres peculiares, nuestra personalidad etc., como medio para conseguir una juventud sin moral, sin sentimientos ni conciencia de pertenecer a un pueblo con unas costumbres discutibles pero indiscutiblemente mejores de las que nos proponen a juzgar por las muestras que vemos (...) no hay duda de que todos los amigos (abiertos o camuflados) de la iglesia utilizan la h de cuya utilización son propugnadores y forzadores. (Arenaza 1974:84-85)

Pero la realidad es que con el paso del tiempo los enemigos del euskera unificado se alejaron de Euskaltzaindia y prevalecieron en ella las ideas de aquellos que hicieron del batúa la prioridad de la Academia. La dirección de la institución fue encomendada a Villasante durante el periodo 1968-1989 en el que, ya definitivamente, se completa el proceso de unificación. Además de regularse la ortografía se emprende la tarea, basándose en las variantes centrales, de unificar el campo más cuajado de diferencias dialectales: la morfología verbal.

\begin{tabular}{|c|c|}
\hline NOR & NOR NORI \\
\hline $\begin{array}{l}\text { - } \\
\text { - } \text { naiz (y no nizeke (y no niteke) } \\
\text { - } \text { zarete }\end{array}$ & $\begin{array}{l}\text { - Zait (y no zaut, zat, jat) } \\
\text { - zitzaidan ( y no zaitan, zautan/jatan) }\end{array}$ \\
\hline NOR NORK & NOR NORI NORK \\
\hline $\begin{array}{l}\text { - } \operatorname{nauzu~(y~no~nazu,~nozu,~nuzu)~} \\
\text { - dut ( y no det, dot) } \\
\text { - dute, naute }\end{array}$ & $\begin{array}{l}\text { - Dit, diot, dizut, digu (y no EUTSI> } \\
\text { deust, deutsatldaut, } * \text { ERADUN> deit/da- } \\
\text { kot, deiot) } \\
\text { - diote } \\
\text { - dizkio ( y no ditio, diotza, deutsaz }\end{array}$ \\
\hline \multicolumn{2}{|c|}{ - Se opta por la vocal más cerrada en el morfema que indica el pasado zinen ( y no zinann) } \\
\hline \multicolumn{2}{|c|}{ 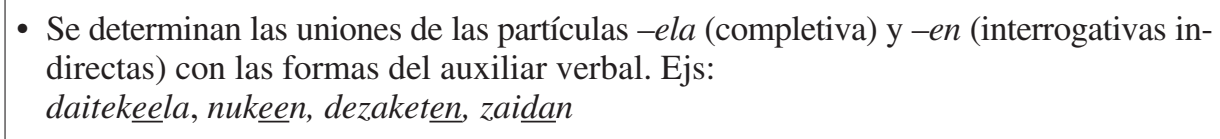 } \\
\hline
\end{tabular}

Estas formas cumplen con los requisitos que debe tener un modelo normativo para ser socialmente aceptado: contaban con el mayor número de hablantes posibles, con una infraestructura que facilitaba su difusión; además gozaban del 
prestigio que les otorgaba la tradición literaria y eran -y son- más comprensibles para quienes no las tienen por variante propia.

Los miembros de la institución contrarios al batúa fundaron asociaciones tales como Euskerazaleak y Kardaberaz Bazkuna en defensa del euskera puro o antiguo que no pudieron oponerse al éxito social del euskera estandarizado y quedaron como residuales -no sin resistencia- en un contexto que asumía el esfuerzo por la estandarización.

Cuando en 1978 la Academia convocó la reunión en Bergara (Gipuzkoa) donde había de evaluar los pasos dados en la última década, no pudo menos que felicitase por los avances conseguidos tanto en la regulación de la lengua como en su implantación social. En esa reunión

- se continuó con la regulación de la ortografía en cuanto a la palatalización (andereño, Maddalen, goxo pero laino, mutila eta ditu)

- se regló, ya definitivamente, el uso de la $<\mathrm{h}>$, se fijaron las desinencias morfológicas para todos los casos de la declinación

- se determinaron, las formas de los demostrativos: hau, hori, hura...

- se determinaron las adaptaciones fonéticas y ortográficas de los préstamos (érradiografia, bake, denbora, gaztelu (pero, RENFE, Sri Lanka, stop, pero penicilina, kontrola en los modernos).

En 1979 se celebró en Arraste-Mondragón (Gipuzkoa) el IX Biltzarra [Congreso]donde se determinó la creación de una nueva comisión, Gramática Batzordea [La Comisión de Gramática]. Sus miembros se han dedicado a estudiar la sintaxis vasca y como resultado de sus esfuerzos se cuenta con Euskal Gramática. Lehen urratsak, es decir, la gramática de referencia de la Academia de la Lengua Vasca cuyo primer volumen se publicó en 1985.

En 1991 (in Euskera 36-2 (1991): 445-446) Euskaltzaindia señala que hay que distinguir entre los préstamos no necesarios y los vocablos que siendo necesarios se eligen del francés o español ahondando más la brecha entre el euskera peninsular y continental (ej.: muntra/erlojua, kotxe/automobila, erretreta-jubilazioa...). La tendencia divergente a nivel léxico entre los hablantes de un lado y otro de la frontera se mantiene hasta hoy y es fuente de reflexión y temores para los estudiosos de la lengua (Sarasola 1997).

En 1994, bajo la convicción de que la normativización facilita y extiende el uso de la lengua, se acordaron las reglas de Euskaltzaindia en las siguientes áreas:

1. Ortografía

2. Variantes lexicales

3. Pronunciación

4. La utilización de guzti+ demostrativo

5. Las formas del hitano (tratamiento de confianza) 
6. Se determina el corpus del euskera batúa desde el año 1995

7. Onomástica

8. Palabras compuestas

No han pasado todavía 10 años desde esa fecha y el euskera estándar es hoy una realidad implantada en la enseñanza y los medios de comunicación. Una vez asumida su necesidad y decidido el punto de partida encauzado por Mitxelena, el proceso ha sido muy rápido y su aceptación social excelente, porque venía a cubrir una carencia que impedía que el euskera se desarrollará con normalidad. Además de las gaueskolas e ikastolas antes mencionadas ha habido otros factores que han sido imprescindibles en la difusión de la lengua estándar y que repasamos aquí:

- El Estatuto de Autonomía (1979)

- La Universidad Pública del País Vasco (UPV-EHU) (1980) y la de Navarra (1898)

- La ley de Normalización y uso del euskera de la CAV y la de Amejoramiento del Régimen Foral que establecía la cooficialidad en la zona norte de Navarra (1982)

- La creación de la EITB (1982), la radio y televisión vasca.

- La regulación de los modelos de enseñanza bilingüe en la enseñanza no universitaria de la CAV (1983) y en Navarra (1988)

- La Secretaría de Política Lingüística del Gobierno Vasco (1983), La ley Foral del Vascuence en Navarra (1986)

- El Plan general de Promoción del uso del euskera en la CAV (1998)

- La existencia del Egunkaria, primer periódico exclusivamente en euskera creado en 1990 y posteriormente Berria (2003)

En el ámbito formal, el euskera estándar, batúa o unificado es la lengua en la que hoy en día se expresan por escrito los vascoparlantes independientemente de su origen geográfico o social. Disponemos de los instrumentos básicos para preservar su naturaleza y función: un conjunto de normas que lo regulan, una gramática descriptiva, Euskal Gramática Lehen Urratsak, y un diccionario del batúa, Hiztegi Batua, y de Autoridades, Orotariko Euskal Hiztegia etc.

Cuando Euskaltzaindia no ha llegado a tiempo para cubrir necesidades perentorias en los medios de comunicación se consagraba el libro de estilo del Egunkaria y el del IVAP (Instituto Público de la Administración Vasca), el corpus contextual de Ereduzko Prosaz gaur, el banco de datos terminológico Euskalterm etc. Son iniciativas que no colisionan con las decisiones de Euskaltzaindia; simplemente responden en su momento a la urgencia de quien traduce, legisla, escribe y nos revelan que la conformación de la lengua común no responde a la postre a una única iniciativa sino que se manifiesta también como una tarea común. 


\section{ÚLTIMAS REFLEXIONES}

El título del Arte de la Lengua Bascongada que el apologista Manuel de Larramendi (1690-1766) publicó en 1729, El imposible vencido, fue una expresión que condensaba la percepción de las dificultades a la hora de sistematizar la estructura del euskera y la sensación de haberla domeñado. Hoy en día la expresión sirve para aplicarla al proceso de estandarización de la lengua vasca pero no así al de la normalización de la misma. A la vista de lo que ocurre con el vasco son dos asuntos que si bien coexisten, no han de correr por fuerza la misma suerte.

Una lengua, si así lo desea la voluntad y el esfuerzo de parte de sus hablantes, puede superar su dispersión dialectal y la fragmentación de sus modelos escritos y dotarse de un modelo de norma culta unificado que garantice su supervivencia. Pero este modelo de lengua será puesto en práctica por la élites intelectuales y no tendrá nunca garantizada su implantación social si no va acompañado de medidas políticas que así lo persigan y que, en definitiva, garanticen tanto la pervivencia del idioma como el derecho lingüístico más fundamental de sus hablantes: la posibilidad de vivir en esa lengua.

Aunque las medidas incluidas en la Ley de Normalización del Euskera en la CAV hayan conseguido el aumento de conocedores del euskera, no está de más recordar aquí que la notabilidad de esos resultados se circunscribe sólo a uno de los tres territorios vascos. La situación en el País Vasco francés -aun a pesar de contar con el euskera batúa modélicamente unificado- no ha mejorado sino que ha ido deteriorándose hasta el punto de peligrar la transmisión intergeneracional.

Por otra parte, finalmente, hay otro hecho que deseo destacar aquí: aunque el índice de bilingües aumenta, parece que el uso que estos hacen de la lengua disminuye ${ }^{20}$. Es decir, conocen la lengua pero la utilizan menos. El euskera se ha convertido para muchos de esos jóvenes bilingües en una lengua limitada al estudio o al trabajo y no la utilizan más allá de estos campos. Habrá quien opine que esta situación es lo suficientemente positiva como para contribuir a la mejora de la situación del vasco pero esto no es así porque no se observan en la sociedad vasca ninguna situación de sesquilingüismo ${ }^{21}$ que nos empuje a compartir esa opinión.

Hay bilingües que no utilizan la lengua porque no son suficientemente competentes de una manera que quisiera mencionar aquí para terminar: el euskera estándar que han aprendido en la escuela, es una lengua de registro culto que no

${ }^{20}$ Una de las conclusiones de la V Encuesta Sociológica (2012) dice así:

$\mathrm{Si}$ en lugar de fijarnos en el estudio del euskera en toda la sociedad, tenemos en cuenta el uso del euskera por parte de los bilingües, la proporción de las personas que utilizan el euskera no ha crecido, a pesar de que haya aumentado el número de bilingües.

${ }^{21}$ Este término propuesto por el profesor Moreno Cabrera define una comunicación en la que cada interlocutor se expresa en el código de su preferencia (Cabrera, 2000). Karlos Cid propone ese sesquilingüismo como un modelo de interlocución que permitiría a los vascoparlantes poder utilizar su lengua en mayor número de ámbitos. 
satisface las necesidades expresivas de los jóvenes. La solución tal vez se encuentre como propone Zuazo (2000) en recurrir a los dialectos para conseguir un euskera más vivo. Si lo desean podemos comenzar el turno de preguntas por esta u otra cuestión.

\section{BIBLIOGRAFÍA}

Arenaza, J. (1974) Tus hijos y el euskera. El mito del batúa. Bilbao, Editorial Vizcaína.

Aresti, G. (1968) «Euskeraren batasuna». Zeruko Argia. Vol. 279, 3.

Baztarrika, P. (2009) Babel o barbarie, Irún, Alberdania.

Brenzinger, M. et al. (2003) Vitalidad y peligro de desaparición de las lenguas. UNES CO. Accesible en http://www.unesco.org/new/fileadmin/MULTIMEDIA/HQ/ CLT/pdf/LVE_Spanish_EDITED\%20FOR\%20PUBLICATION.pdf

Moreno Cabrera, J. C. (2000) La dignidad e igualdad de las lenguas. Crítica de la discriminación lingüística, Madrid, Alianza Editorial.

Cid, C. (2002) «Las fronteras de la lengua vasca a lo largo de la historia», Revista de Filología Románica, 19, Madrid, Universidad Complutense. Accesible también en revistas.ucm.es/index.php/RFRM/article/download/.../10736

Crystal, D. (2005) La revolución del lenguaje, Madrid, Alianza Editorial.

Crystal, D. (2001) La muerte de las lenguas, Madrid, Cambridge University Press.

Gobierno Vasco (2008) IV Encuesta Sociolingüística. 2006: Comunidad Autónoma Vasca, País Vasco Norte, Navarra, Euskal Herria.Vitoria-Gasteiz, Gobierno Vasco. Accesible también en

http://www.kultura.ejgv.euskadi.net/r46-17894/es/contenidos/informacion/argitalpenak/es_6092/adjuntos/IV_incuesta_es.pdf

Gobierno Vasco (2012) Resumen V Encuesta Sociolingüística 2012. Comunidad Autónoma Vasca, País Vasco Norte, Navarra, Euskal Herria. Accesible también en http://www.kultura.ejgv.euskadi.net/r46-17894/es/contenidos/informacion/argitalpenak/es_6092/adjuntos/EHResumen_encuesta_2011.pdf

http://www.euskera.euskadi.net/r59738/es/contenidos/informacion/argitalpenak/es_6092/i kuspegi_sozio_linguis.html

Menénez Pidal, R. (1962) «Introducción al estudio de la lingüística vasca», En torno a la lengua Vasca, Buenos Aires, Espasa Calpe.

Mitxelena, K. (1988) «Euskeraren batasun bideak orain», ASJU, Sobre Historia de la Lengua Vasca. Vol II, 972-983. Donostia, Universidad del País Vasco-Euskal Herriko Unibertsitatea.

Moseley, Ch. (ed.). (2010). Atlas de las lenguas del mundo en peligro, $3^{a}$ edición, Ediciones UNESCO. París.Versión en línea: http://www.unesco.org/culture/languagesatlas/es/atlasmap.html

Sarasola, I. (1976) Historia social de la literatura vasca, Akal, Madrid.

- 1997) Euskera batuaren ajeak, Irun, Alberdania. 
Torrealdai, J. M. (1998) El libro negro del euskera, Donostia-San Sebastián, Tártalo.

Txillardegi (2004) Euskeraren aldeko borroka, Donostia-San Sebastián, Elkar.

Villasante, L. (1980) Serie Luis de Eleizalde sobre la unificación del euskera escrito, vol. I, Hacia la lengua literaria común, Oñate, Editorial franciscana Arantzazu.

Ugalde, M. et al. (1984) Euskaltzaindiaren historia laburra. Bilbo. Euskaltzaindia.

Villasante, L. (1988) Serie Luis de Eleizalde sobre la unificación del euskera escrito, vol. IX: Euskeraren auziaz, Oñate, Editorial franciscana Arantzazu.

Zuazo, K. (2000) Euskeraren sendabelarrak, Irun, Alberdania.

Zuazo, K. (2005) Euskera Batua. Ezina ekinez egina, Donostia, Elkar. 